\title{
Study of systemic chromatic changes in color space to model color transparency
}

\author{
Peggy Gérardin ${ }^{a}$, Sabine Süsstrunk ${ }^{a}$ and Kenneth Knoblauch ${ }^{b}$ \\ ${ }^{a}$ Laboratory for Audiovisual Communications, Swiss Federal Institute of Technology (EPFL), \\ Switzerland \\ ${ }^{b}$ Institut National de la Santé et de la Recherche Médicale U371 (INSERM), 69675 cedex \\ Bron, France
}

\begin{abstract}
Several studies have suggested that translation and convergence in a linear trichromatic color space lead to the perception of transparency, but other transformations, such as shear and rotation, do not. We have designed a psychophysical experiment to study the limits of such systemic chromatic changes, adding categories such as different luminance levels and vector lengths. The number of our stimuli and the number of observations provide strong statistical support for D'Zmura's model. Our main results show that for vectors exceeding a minimal length, convergence and translation (except in the equiluminant plane) lead to the perception of transparency, while shear and divergence do not. However, our results reveal that small shears and divergences also appear transparent. We also found that large translations in the equiluminant plane tend to be less often judged as transparent.
\end{abstract}

Keywords: Color transparency, phsychophysics, systemic chromatic changes, equiluminant plane, vector length, log-linear model.

\section{INTRODUCTION}

Color transparency provides an example for a color constancy phenomenon. ${ }^{1,2}$ A surface seen at the same time through a transparent colored filter and directly is identified as one and unique surface, independant of the illuminant variations that occur. The observer separates chromatic properties of the transparent filter (and defines the color of the filter) from those of the surface that is under the filter. The interpretation of surface cues requires a global integration of the image's local characteristics.

Transparency can occur even without color changes. According to Metelli, ${ }^{3}$ three conditions are needed to perceive transparency with overlapping surfaces: the uniformity of the transparent layer, the continuity of its bounderies and an adequate stratification. Indeed, when the unity of the central region is broken, the perception of transparency is lost. To understand better the perception of transparency, researchers look for conditions that produces this phenomenon, such as luminance relations ${ }^{3-7}$ or spatial configuration. ${ }^{8,9}$ For example, an Xjunction seems to be a good indicator for transparency. If two surfaces overlaid by a filter give an intersection between four different regions, they might be interpreted by the human visual system as transparent.

The role of color has also been explored. ${ }^{10-12}$ For example, the observations of D'Zmura et al. ${ }^{13}$ suggest that chromatic changes such as translations and convergences in the CIE color space give the impression of transparency.

The goal of this study is to determine the limits of such systemic chromatic changes in generating them with a simpler spatial configuration than was used in the original study, ${ }^{13}$ and analyzing the results with respect to luminance level and vector length variations.

Further author information: (Send correspondence to Peggy Gérardin)

E-mail: Peggy.Gerardin@epfl.ch, Telephone: +41216936609 


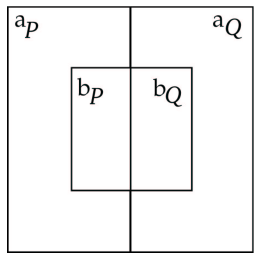

Figure 1. Example of a bipartite field containing only two X-junctions: when we apply a translation or a convergence in a linear trichromatic color space to the surfaces $\mathbf{a}_{P}$ and $\mathbf{a}_{Q}$, we obtain a transparent effect displayed with surfaces $\mathbf{b}_{P}$ and $\mathbf{b}_{Q}$.

\section{THE CONVERGENCE MODEL}

D'Zmura et al. ${ }^{13}$ developped a model of color transparency perception based on additive color mixtures. They suggested that translation and convergence in CIE XYZ space lead to the perception of transparency, but not shear and rotation. They formulated translation in CIE XYZ as

$$
\mathbf{b}_{P, Q}=\mathbf{a}_{P, Q}+\mathbf{t}
$$

where $\mathbf{a}=\left(X_{\mathrm{a}} Y_{\mathrm{a}} Z_{\mathrm{a}}\right)$ represents the tristimulus values of a surface, and $\mathbf{t}=\left(X_{\mathrm{t}} Y_{\mathrm{t}} Z_{\mathrm{t}}\right)$ a translation that is added to the surface $\mathbf{a}$ to provide new tristimulus values $\mathbf{b}=\left(X_{\mathrm{b}} Y_{\mathrm{b}} Z_{\mathrm{b}}\right)$.

They also proposed an equation for convergence (equ. (2)), where tristimulus values $\mathbf{a}_{P}$ and $\mathbf{a}_{Q}$ represent surfaces and the target of convergence is represented by a vector of tristimulus values $\mathrm{g}=\left(X_{\mathrm{g}} Y_{\mathrm{g}} Z_{\mathrm{g}}\right)$. The transparent overlay is charcaterized by vectors $\mathbf{b}_{P}$ and $\mathbf{b}_{Q}$ that are located on lines connecting $\mathbf{a}_{P}$ and $\mathbf{a}_{Q}$ to $\mathbf{g}$, respectively. The result for the vectors $\mathbf{b}_{P}$ and $\mathbf{b}_{Q}$ can be written as:

$$
\mathbf{b}_{P, Q}=(1-\alpha) \mathbf{a}_{P, Q}+\alpha \mathbf{g}, \quad \text { with } \quad 0<\alpha<1
$$

The parameter $\alpha$ represents the distance of $\mathbf{b}_{P, Q}$ from $\mathbf{a}_{P, Q}(\alpha=0)$ to $\mathbf{g}(\alpha=1)$. The authors stress the fact that convergence of the lights from many surfaces towards a single point provides a systemic chromatic change that is interpreted by the visual system as transparency. D'Zmura et al. ${ }^{2,14}$ found that combinations of translation and convergence also produces transparency. Thus, they proposed a model combining both, referred to as the Convergence Model (translation can be viewed as convergence to a point at infinity), written as

$$
\mathbf{b}_{P, Q}=(1-\alpha) \mathbf{a}_{P, Q}+\alpha \mathbf{g}+\mathbf{t}, \quad \text { with } \quad 0<\alpha<1
$$

They examined whether this model describes the color appearance of surfaces seen behind transparent filters. They tested the Convergence Model psychophysically ${ }^{14}$ and also compared it to other models such as the von Kries model. ${ }^{2}$ They found that their model better describes the colors seen behind transparent filters. They have also found that it describes well the color appearance of surfaces seen through fog, since this model takes into account color shift and change in contrast. ${ }^{15}$

As the Convergence Model is psychophysically well tested, we wanted to evaluate the limits of chromatic changes, such as translation, shear, divergence and pure convergence $(\mathbf{t}=0)$, as well. We tested each condition for different luminance levels and with different vector lengths. Our study underlines the role of contrast changes and its close relationship with vector length. 


\section{METHOD}

\subsection{Equipment}

The stimuli were displayed on a BARCO monitor connected to a DELL (Precision 6.1.0) PC (the resolution was $1280 \mathrm{x} 1024,256$ colours, and the frame rate was $75 \mathrm{~Hz}$ ). After the monitor calibration (GretagMacbeth Eye-One Monitor), the spectral radiance of the monitor was measured with a Minolta CS-1000 spectroradiometer to obtain the gamma of the monitor and then to find the best correspondance between linearized monitor RGB and 1931 CIE XYZ.

\subsection{Stimuli}

The experimental stimuli were created with MATLAB v.6.0.088 R12, ${ }^{16}$ and displayed with WINVIS. ${ }^{17}$ The stimuli consisted of a bipartite field (10x10 deg.) partially overlaid by a simulated square $(5 \times 5$ deg.), displayed at the center of the monitor. A total of 240 stimuli were selected: 60 per each chromatic change, 20 per each luminance level and 2 per each vector length. Four types of color change were tested: pure translation (equ. (1)), pure convergence (equ. (2)), shear, and divergence (with $\varphi=\pi / 4$ of one vector divergence) in the CIE $\mathrm{XYZ}$ space created as followed, respectively:

$$
\begin{gathered}
\mathbf{b}_{P}=\mathbf{a}_{P}+\mathbf{t} \quad \text { and } \quad \mathbf{b}_{Q}=\mathbf{a}_{Q}-\mathbf{t} \\
\mathbf{b}_{P}=\mathbf{a}_{P}+\mathbf{t} \quad \text { and } \quad \mathbf{b}_{Q}=\left(\mathbf{a}_{Q}+\mathbf{t}\right)+\varphi
\end{gathered}
$$

Three conditions accounted for luminance: vectors in the equiluminant plane, vectors that point to a lower luminance (filter) and vectors directed to a higher luminance (illumination). Ten different vector lengths were assigned to these conditions, with $\|\mathbf{t}\|=\{1,2, \ldots, 10\}$ for translations, shears, and divergences, and $\alpha=\{0.1,0.2, \ldots, 1\}$ for convergences.

\subsection{Procedure}

Six (two female and four male) observers were selected for their normal or corrected-to-normal visual acuity and normal color vision, according to the Farnsworth-Munsell 100-Hue-Test. The psychophysical experiments took place in a completely dark room. Subjects sat in front of the monitor at $50 \mathrm{~cm}$ from the screen. The set of all patches was presented in a randomized sequence. For each patch, the subjects responded (with pre-established keyboard commands) whether they saw a transparent overlay or not, or whether they did not know. Each stimulus was shown for two seconds. The next trial was not presented until the subject responded. The session containing the 480 stimuli was run four times. A training set of ten stimuli was presented before each session and discarded. No feedback was provided during the experiment.

\section{RESULTS}

We collected the observers' responses in a matrix containing every chromatic change (translation, convergence, shear and divergence), every luminance type (equiluminant, filter and illumination), and the ten different vector lengths. A Log-Linear Model ${ }^{18}$ was used to calculate the relationship between the different variables. We evaluated the model fitted to the data with the Pearson chi-square statistics $\left(\chi^{2}\right)$.

We first expect to find an interaction between response type and the type of chromatic shift ('transparent' related to convergence and translation, and 'not transparent' related to shear and divergence). Then, we can also conceive of an association between subjects' responses and other variables such as luminance levels and vector length. We have found the following model that describes the data as well as the saturated model:

$$
A+A * S+A * L+S * L+A * S * D+A * S * L+S * D * L
$$

where $A$ is the response type, $S$ is the type of chromatic change (convergence, translation, shear and divergence), $D$ is the luminance level (equiluminant plane, illuminant or filter) and $L$ represents the vector lengths. 

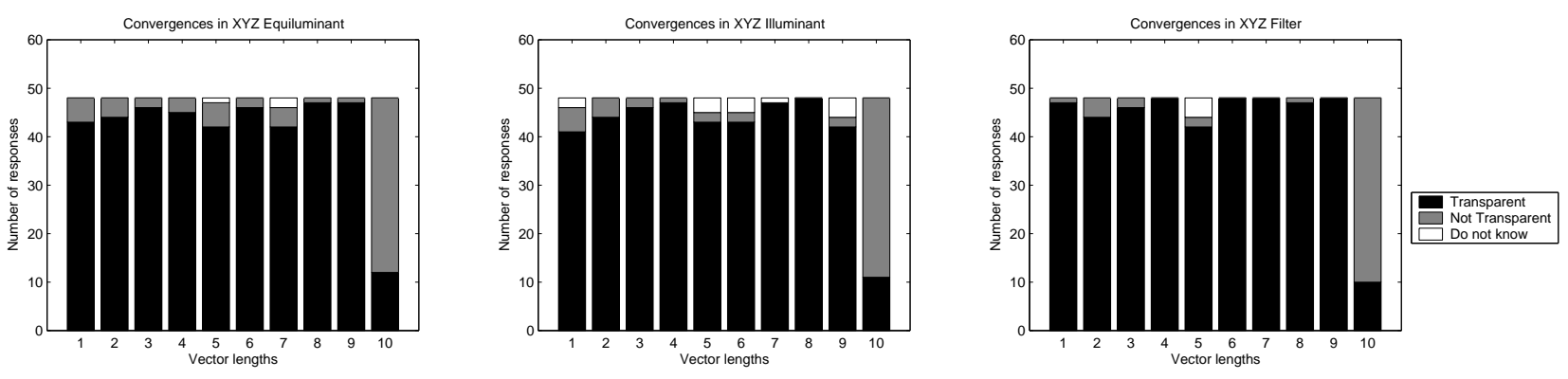

Figure 2. These figures show the frequency for subjects' responses for convergences in equiluminant (a), illuminant (b), and filter (c) categories.

We observe that all terms are not necessary to describe the data, and only response type $A$ must be added to the model. We then notice several interactions that depict the data.

As expected, we discern an interaction between response type $A$ and type of chromatic change $S$. Thus, we have different responses according to the chromatic shift. We also see an interaction between response type and vector length $L$, and another one between type of chromatic change $S$ and vector length $L$. A more complex interaction between response type $A$, chromatic change $S$, and luminance type $D$ is also observed. We denote the same interaction with vector length $L$ instead of luminance type. Finally, we remark an interaction between the type of chromatic shift, the luminance type and vector length. In the following paragraphs, we discuss each type of chromatic change (fig. 2-5) according to the interactions found in our model.

Convergence The large number of stimuli presented (chosen randomly in the XYZ space) and the number of observations provide strong statistical evidence in support of the D'Zmura et al. ${ }^{2,14}$ Convergence Model. We present in figure 2 the number of subjects' responses for each color shift, classified in three response types: 'transparent', 'not transparent', 'do not know'. In general, observers detected transparency very well as long as the stimuli were generated by this model. Subjects perceived transparency independent of luminance category or vector length. The last column in each figure shows a majority of 'not transparent' responses, due to $\alpha=1$ (opaque overlay). Thus, responses are constant among all presented stimuli.

Translation As figure 3 shows, subjects detect transparency well with stimuli produced by translations to illuminant and filter-like luminance changes. No vector length effect is observed. However, results for detecting a transparent overlay produced by equiluminant color changes are less straight-forward (fig. 3 (a)). The results suggest that for large vector lengths, the transparency is less evident than for short vector lengths. Thus, they do not see a transparent overlay. It presupposes that detection of transparency depends not purely on translation, but also on luminance category combined with vector length, as it is shown in our model (equ. (6)). Results in the equiluminant plane may be described by interactions between responses $A$ with chromatic shifts $S$ and luminance category $D$, as well interactions between color changes $S$ with luminance $D$ and vector length $L$. Transparency perception depends on translations directed to low and high luminance levels, and on equiluminant translations with short vector length.

Shear The results for shear are intriguing (fig. 4). It appears that subjects detect transparency for small shears, especially in the equiluminant plane (fig. 4 (a)), whereas large shears do not appear transparent, as expected. The first figure shows a propensity to detect transparency with shorter vector lengths in the equiluminant plane. The model (equ. (6)) expresses such a result, showing an interaction between response type $A$ and vector length $L$. It appears that some color change (here, shear) interacts with vector length $(S * L)$. It also specifies interactions between response type $A$ with type of chromatic change $S$ and vector length $L$, and interactions between type of color shift $S$ with luminance $D$ and vector length $L$. The last figures show responses for illuminant and filter 

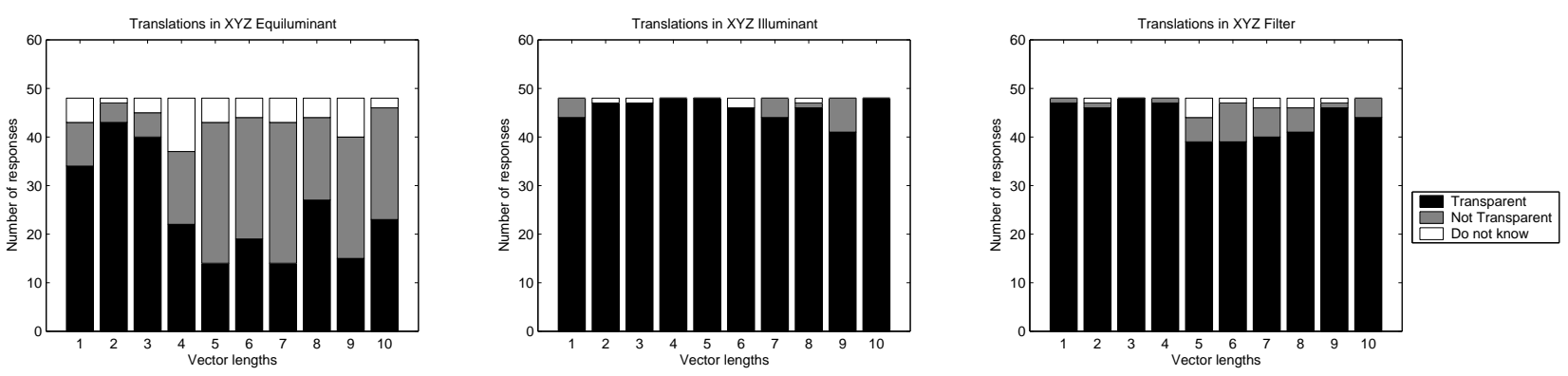

Figure 3. These figures show the frequency for subjects' responses for translations in equiluminant (a), illuminant (b), and filter (c) categories.
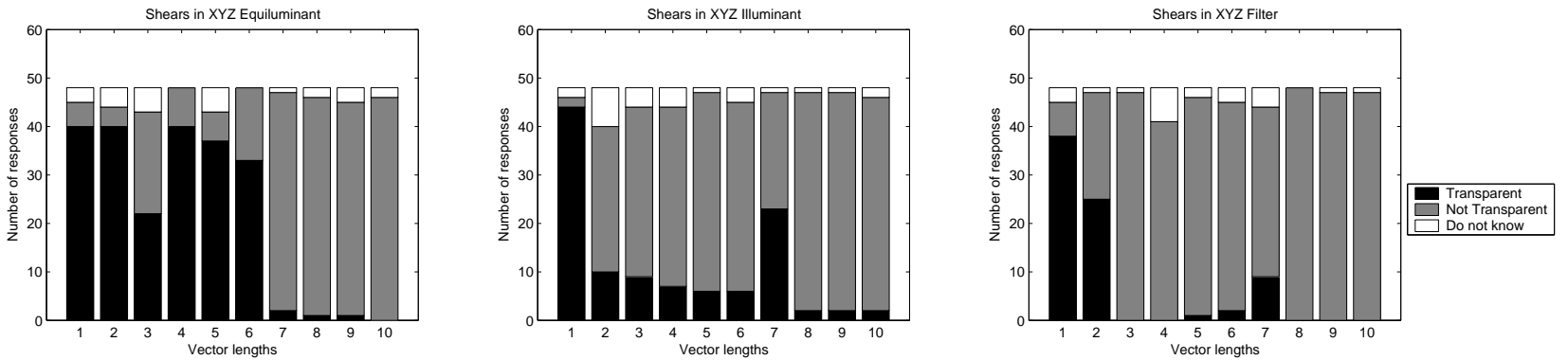

Figure 4. These figures show the frequency for subjects' responses for shears in equiluminant (a), illuminant (b), and filter (c) categories.

categories (fig. $4(\mathrm{~b}, \mathrm{c})$ ). Short vector lengths generate transparency $(\|\mathbf{t}\|=1$ ), while stimuli with $\|\mathbf{t}\|>2$ tend to appear opaque.

Divergence Observers tend to respond 'not transparent' when the stimuli are created by larger divergent vectors (fig. 5). However, they detect transparency for short divergences in every luminance condition (equiluminant, illuminant and filter), opposite to shear, where a large number of equiluminant shears lead to the perception of transparency. As our model specified, response type $A$ clearly interact with vector length $L(A * L)$, as well as chromatic change $S$ interacts with luminance $D$ and vector length $L(S * D * L)$. As for shear, short vector lengths lead to the perception of transparency, in each luminance category, with vector length $\|\mathbf{t}\| \leq 2$.
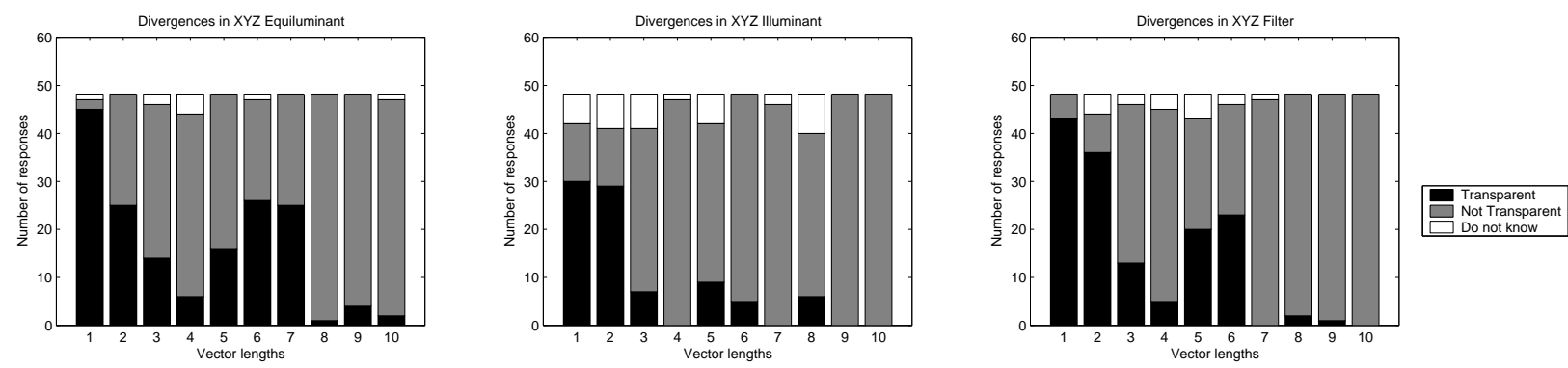

Not Transparent 作

Figure 5. These figures show the frequency for subjects' responses for divergences in equiluminant (a), illuminant (b), and filter (c) categories. 


\section{DISCUSSION}

Studies ${ }^{2,13,14}$ suggest that chromatic changes that induce perceived transparency only include convergence and translation. The convergence model has been well tested, ${ }^{14}$ and appears to work generally for transparency perception. In previous work, observers had to adjust stimuli to attain transparency. Our study provides much more possibilities to subjects since they have to judge transparency of independently chosen stimuli, according to vector length and luminance conditions. In our results, convergences, despite luminance and vector length variations, appear transparent. However, large equiluminant translations do not appear transparent. Also, small shears and divergences produce transparency.

Our results suggest that these changes are not equal across luminance level variations: we have shown that subjects have more difficulties seeing transparency based on equiluminant translations than on equiluminant convergences. Our data provide the same observation as these previous studies, showing that convergence produces the most positive responses for transparency, despite any luminance variations. Nevertheless, observers do not seem to see transparency for all equiluminant translations. Longer length vectors show less transparency. Luminance is one of necessary parameters for translation, and vector length also. When we present equiluminant translations to the subject, they appear transparent depending on vector length. We have found that convergence does not give such a result, probably because all our vectors are directed to the same hue. This might create a sufficient perceptual link between the overlay and the original surface. However, it was found by Chen et al. that the Convergence Model does not lead to the perception of transparency when surface colors have opposite hues or lightness. ${ }^{14}$ We assume that, for translations, when the vector based at a given hue exceeds a certain locus around this hue (e. g. directed to an opposite hue), the chromatic contrast is too large to give the impression of transparency.

We wanted to test whether other chromatic changes, such as shear and divergence, do not give the perception of transparency. Nevertheless, our results also show that for short vector lengths, observers do detect transparency with shear and divergence. We can suppose $\mathrm{X}$-junctions are sufficient cues to perceive transparency under these conditions, and that for small chromatic changes, this is enough to generate perceptual scission, despite any particular chromatic change. Small shears or divergences tested using an L-junction configuration could evaluate this hypothesis.

\section{CONCLUSION}

We have strong statistical evidence that translations and convergences lead to the perception of transparency, and shears and divergences do not, with some constraints. D'Zmura et al. ${ }^{13}$ showed that equiluminant translations and convergences lead to such a result, and so substractive (filter) and additive (illumination) models are not the only conditions for perceiving transparency. We have seen that this is true for convergences. On the other hand, large translations in the equiluminant plane do not clearly appear transparent, in contrast to large equiluminant convergences. We have also shown that for small vector lengths, shear and divergence appear transparent. Further research is needed to clarify the relations between systemic chromatic changes with luminance, and the spatial configuration of the stimulus (tests with L-junction configurations, for example).

\section{ACKNOWLEDGMENTS}

This work was supported by the Swiss National Science Foundation under grant number 21-59038.99.

\section{REFERENCES}

1. K.-H. Bauml, "Simultaneous color constancy: how surface color perception varies with the illuminant," Vision Research 39, pp. 1531-1550, 1999.

2. M. D'Zmura, O. Rinner and K. R. Gegenfurtner, "The colors seen behind transparent filters," Perception 29, pp. 911-926, 2000.

3. F. Metelli, "The perception of transparency," Scientific American 230, pp. 90-98, 1974.

4. J. Beck, "Additive and substractive color mixture in color transparency," Percept. Psychophys. 23, pp. 265$267,1978$. 
5. J. Beck, K. Prazdny and R. Ivry, "The perception of transparency with achromatic colors," Percept. Psychophys. 35, pp. 407-422, 1984.

6. W. Gerbino, C. I. Stultiens, J. M. Troost and C. M. de Weert, "Transparent layer constancy," J. Exp. Psychol. Hum. Percept. Perform 16(1), pp. 3-20, 1990.

7. K. Nakayama, S. Shimojo and V. Ramachandran, "Transparency: relation to depth, subjective contours, luminance and neon color spreading," Perception 19, pp. 497-513, 1990.

8. E. H. Adelson, "Perceptual organization and the judgment of brihtness," Science 262, pp. 2042-2044, 1993.

9. T. Watanabe and P. Cavanagh, "Transparent surfaces defined by implicit x-junctions," Vis. Res. 33(16), pp. 2339-2346, 1993.

10. O. D. Pos, Trasparenze, Icone s.r.l., first ed., 1989.

11. F. Faul, "Chromatic scission in perceptual transparency," Perception 25(Supplement), p. 105, 1996.

12. F. Faul and V. Ekroll, "Psychophysical model of chromatic perceptual transparency based on substractive color mixture," J. Opt. Soc. Am. 19(6), pp. 1084-1095, 2002.

13. M. D'Zmura, P. Colantoni, K. Knoblauch and B. Laget, "Color transparency," Perception 26, pp. 471-492, 1997.

14. V. Chen and M. D'Zmura, "Test of a convergence model for color transparency," Perception 27, pp. 595-608, 1998.

15. J. Hagedorn and M. D'Zmura, "Color appearance of surfaces viewed through fog," Perception 29, pp. 1169$1184,2000$.

16. Mathworks (Natick), MA., U.S.A.

17. Neurometrics (Berkeley), CA., U.S.A.

18. D. Knoke and P. Burke, Log-Linear Models, vol. 20, Sage University Paper, 1980. 